\title{
Acamprosate Complements Ill-Sustained Response of ECT in Catatonic Fronto- temporal Dementia
}

\author{
Ahmed Naguy $^{1 *}$, Ali Al-Tajali ${ }^{2}$ and Haya al-Mutairi ${ }^{3}$ \\ ${ }^{1}$ Child and Adolescent Psychiatrist Centre for Mental Health, Kuwait \\ ${ }^{2}$ General Adult Psychiatrist of Neuromodulation Unit, KCMH, Kuwait \\ ${ }^{3}$ Consultant Psychiatrist Hospital and Director General of KCMH, Kuwait
}

Keywords: Frontotemporal dementia; Catatonia; ECT; Acamprosate

\section{To The Editor}

It is estimated that $10-15 \%$ of demented patients have clinical characteristics suggestive of Fronto-temporal dementia (FTD) or Pick's disease. It is the third most common neurodegenerative cortical dementia after Alzheimer's disease and diffuse Lewy's Body disease. It equally affects both males and females, within the age group of 45-65 years. It manifests mainly as insidious onset of behavioral and personality changes. MRI characteristically shows parietal and anterior temporal lobar atrophy. Psychiatric aspects seem to respect the patterns of classical frontal lobe syndromes. Speech and language abnormalities often begin early and progress rapidly. Incontinence can occur early. Parkinsonism could be an association too. Examination is usually remarkable for witzelsucht, echo phenomena, primitive reflexes, gegenhalten, logopenia, anomia, perseverations but with relatively spared visuo-spatial skills and eventually lack of insight $[1,2]$. Catatonia, coined historically by Kahlbaum, could complicate organic, affective, schizophrenic, autistic and demented presentations and comprise a constellation of motor phenomena including, inter alia, automatic obedience, ambitendency, catalepsy, gelatio, cerea flexibilitas, echolalia, echopraxia, psychogenic pillow, mannerism, mitmachen, mitgehen, stereotypy, negativismus and stupor, only to mention a few $[3,4]$. Most experts agree that catatonia is exceedingly underdiagnosed in neuropsychiatric community. This is especially true in the case of FTD, where clinical distinction between both could be too arduous in which cases therapeutic testing with lorazepam or even ECT could be diagnostic $[5,6]$. It should be borne in mind; however, that catatonia and dementia are not mutually exclusive diagnoses and neurologic conditions associated with catatonia are protean including herpetic encephalitis, frontal lobe trauma, paraneoplastic, epilepsy, syphilis and temporal lobe infarction [7]. Prevalence of catatonia in dementia was circa $15 \%$ in a study from Scotland [8]. Cardinal catatonic features commonly overlap with advanced dementia [9]. Case reports of this composite presentation abound in the literature [10,11]. Even iatrogenic cases, due to the use of anti-dementia drug donepezil, were also reported [12]. It seems sensible that treating the catatonic component of a patient's dementing process may ameliorate a reversible or modifying factor and we opine that patients should never be denied this opportunity, if any as the impact in toto could be tremendous [13]. For such puzzling cases of catatonic demented patients, unfortunately, there is no roadmap to guide clinicians as regards the most appropriate course of action in these scenarios. Neurobiologic underpinngs of catatonia basically involves cortical GABA deficiency and glutamate excitotoxicity [14]. Benzodiazepines and electro-convulsive therapy (ECT) are the treatment modalities of choice in catatonia irrespective of underlying causation [15]. Regrettably, clinicians are often-time skeptical and reluctant to pursue with this ostensibly "aggressive" manoeuvres in "frail" demented patients. Acamprosate is anti-craving agent in alcoholism with a putative NMDA-gluatmate antagonistic activity and GABAa enhancing activity [16]. In theory, it neatly fits with neurobiologic model of catatonia. Having said so, we report the following case of catatonic FTD that responded initially to ECT but this was short-lived ill-sustained response where addition of acamprosate resulted in well-sustained outcome. Interestingly, this was achieved with high tolerability and safety given that acamprosate has no effect on hepatic microsomal system.

A 59-year-old Kuwaiti male, long diagnosed as FTD, with a residential placement in Psycho-geriatrics Unit, a known controlled hypertensive, maintained on sertraline $50 \mathrm{mg} / \mathrm{d}$ to help obvious apathy, was noted to be progressively psychomotorically sluggish, almost totally mute, resistive, negativistic, occasional stereotypies and paratonia on exam. Diagnosis of superimposed catatonia was entertained. A therapeutic test with IM lorazepam was positive. A course of ECT was suggested and discussed with care-givers. After 5 sessions of modified bitemporal ECT, a tangible improvement was noted but unfortunately, ill-sustained. We splitted over pursuing further with ECT or employing high-dose lorazepam strategy but both were detracting in terms of cognitive drawbacks and past history of pulmonary embolism for the latter. We thought of embarking on a trial with acamprosate for its pharmacologic portfolio and benign side-effects as well. Caregivers consent was obtained beforehand. We dosed it at $333 \mathrm{mg}$ tds. Over next few days, catatonia markedly abated and response was well-sustained. This was achieved with high tolerability. After a couple of weeks, we attempted at withdrawing acamprosate, patient readily slipped into catatonoid state that resolved rapidly when acamprosate was reinstituted at same dosage. The patient fared well over next 6 months. Buch-Francis Catatonia Rating Scale at baseline (read 39) and followups (dropped to 8 ) was used all through to objectify clinical findings.

To our knowledge, this is one of first cases reporting utility of acamprosate in catatonia, which could open new venues for treatment when first-line treatments are ineffective or intolerable especially in geriatric population with already compromised cognitions. And certainly, well-designed trials are needed to draw definite conclusions about the use of acamprosate in catatonia.

*Corresponding author: Ahmed Naguy, Kuwait Centre for Mental Health $(\mathrm{KCMH})$, State of Kuwait, Kuwait, Tel: 96565541937; E-mail: ahmednaguy@hotmail.co.uk

Received March 27, 2015; Accepted April 21, 2015; Published April 27, 2015

Citation: Naguy A, Al-Tajali A, Al-Mutairi H (2015) Acamprosate Complements IIISustained Response of ECT in Catatonic Fronto-temporal Dementia. J Psychiatry 18: 292 doi: $10.4172 / 2378-5756.1000292$

Copyright: ( 2015 Naguy A, et al. This is an open-access article distributed under the terms of the Creative Commons Attribution License, which permits unrestricted use, distribution, and reproduction in any medium, provided the original author and source are credited 
Citation: Naguy A, Al-Tajali A, Al-Mutairi H (2015) Acamprosate Complements III-Sustained Response of ECT in Catatonic Fronto-temporal Dementia. J Psychiatry 18: 292 doi: 10.4172/2378-5756.1000292

Page 2 of 2

\section{Disclosures}

Authors declare no conflicts of interest.

\section{References}

1. Warren JD (2013) Fronto-temporal dementia. BMJ 347: f4827.

2. Snowden J, Neary D, Mann D (2002) Frontotemporal dementia. British Journal of Psychiatry 180: 140-143.

3. Naguy A (2013) 'Catatonia' in Schizophrenia; a Nifty Compendium. 2nd Edn. Alex Press 45-49.

4. Carroll B, Kennedy J, Goforth H (2000) Catatonic signs in medical and psychiatric catatonias. CNS Spectr 5: 66-69.

5. Suzuki K, Takano T, Matsuoka H (2009) A case of catatonia resembling frontotemporal dementia and resolved with elecrtroconvulsive therapy. World J Biol Psychiatry 10: 245-247.

6. Lauterbach E, Kuppuswamy P, Greenway L (2010) Differential pharmacological responses of catatonia-like signs in frontotemporal dementia. Neurocase: The Neural Basis of Cognition 16: 436-450.

7. Fink M, Taylor M (2003) Catatonia: A clinician's guide to diagnosis and treatment. Cambridge University Press, New York 98.
8. Al-Sayegh A, Reid D (2010) Prevalence of catatonic signs in acute psychiatric patients in Scotland. BJPsych Bulletin 34.

9. Eisenstock $J(2010)$ On catatonia and dementia: a case report. Neurol Bull 2:12-16.

10. Kendurkar A (2008) Catatonia in an Alzheimer's dementia patient. Psychogeriatrics 8: 42-44.

11. Holm A (2014) Neurodegenerative and psychiatric overlap in frontotempora lobar degeneration: a case of familial frontotemporal dementia presenting with catatonia. International Psycogeraitrics 26: 345-347.

12. Morita S, Miwa H, Kondo T (2004) A patient with probable Lewy bodies who showed catatonia induced by donepezil: a case report. Brain and Nerve 56 : 881-884.

13. Alisky JM (2004) Is the immobility of advanced dementia a form of lorazepamresponsive catatonia? Am J Alzheimers Dis Other Demen 19: 213-4.

14. Daniels J (2009) Catatonia: clinical aspects and neurobiological correlates. Psychiatryonline 21: 371-380

15. Rajagopal S (2007) Catatonia. Advances in Psychiatric Treatment 13: 51-59.

16. Kufahl PR, Watterson LR, Olive MF (2014) the development of acamprosate as a treatment against alcohol relapse. Expert Opin Drug Discov 9: 1355-1369. 